\title{
Nonuniqueness of algebraic first-order density-matrix functionals
}

\author{
Jian Wang* \\ School of Science, Huzhou University, Zhejiang 10083, China \\ Peter J. Knowles ${ }^{\dagger}$ \\ School of Chemistry, Cardiff University, Main Building, Park Place, Cardiff CF10 3AT, United Kingdom \\ (Received 4 May 2015; published 27 July 2015)
}

\begin{abstract}
By explicit construction of counterexamples having the same eigenvalue spectrum of one-matrix, but different two-matrix, we show that density-matrix functionals for the electronic energy that are based solely on the eigenvalues of the one-matrix cannot be unique in functional representation of the two-matrix. The one-to-many mapping may be understood either through the number of independent parameters or the contraction relation.
\end{abstract}

DOI: 10.1103/PhysRevA.92.012520

PACS number(s): 31.15.ec, 31.15.ve, 31.10.+z, 31.15.A-

The one-particle reduced density matrix, or one-matrix, is defined as

$$
\gamma\left(\mathbf{x}, \mathbf{x}^{\prime}\right)=\sum_{i j}\left\langle\Psi\left|a_{i}^{\dagger} a_{j}\right| \Psi\right\rangle \phi_{i}^{*}(\mathbf{x}) \phi_{j}\left(\mathbf{x}^{\prime}\right)
$$

where $\mathbf{x}$ stands for both the spatial (r) and the spin (s) coordinates, and $a_{i}^{\dagger}$ and $a_{j}$ are the creation and annihilation operators for the one-particle basis $\left\{\phi_{i}\right\}$ that generates an $N$-particle product space in which the wavefunction $\Psi$ lives. Since the one-matrix is Hermitian, one can always diagonalize it and obtain its spectral form $\gamma\left(\mathbf{x}, \mathbf{x}^{\prime}\right)=\sum_{i} n_{i} \chi_{i}^{*}(\mathbf{x}) \chi_{i}\left(\mathbf{x}^{\prime}\right)$. The eigenvectors $\left\{\chi_{i}\right\}$ are called the natural orbitals, and the eigenvalues $\left\{n_{i}\right\}$ are their occupation numbers.

Similarly the two-particle reduced density matrix, or twomatrix, can be defined as

$$
\begin{aligned}
\Gamma\left(\mathbf{x}_{1}, \mathbf{x}_{2}, \mathbf{x}_{1}^{\prime}, \mathbf{x}_{2}^{\prime}\right) & =\sum_{i j k l} \Gamma_{i j, k l} \phi_{i}^{*}\left(\mathbf{x}_{1}\right) \phi_{j}^{*}\left(\mathbf{x}_{2}\right) \phi_{k}\left(\mathbf{x}_{1}\right) \phi_{l}\left(\mathbf{x}_{2}\right), \\
\Gamma_{i j, k l} & =\left\langle\Psi\left|a_{i}^{\dagger} a_{j}^{\dagger} a_{l} a_{k}\right| \Psi\right\rangle .
\end{aligned}
$$

Using natural orbitals, the exact energy of an electronic system can be written as

$$
E=Z+\sum_{i} h_{i i} n_{i}+\frac{1}{2} \sum_{i j k l} \Gamma_{i j, k l}\langle i j \mid k l\rangle,
$$

where $h_{i j}$ and $\langle i j \mid k l\rangle$ are the usual one- and two-electron integrals in the natural orbital basis, and $Z$ is the bare-nucleus Coulomb energy. In reduced density-matrix functional theory (RDMFT) [1], one tries to model the two-electron part of the potential energy by representing the two-matrix $\Gamma$ as a functional of the one-matrix (or of, equivalently, the natural orbitals and their occupation numbers). Note that in contrast to Kohn-Sham density-functional theory, all of the kinetic energy is represented exactly. Commonly used functionals assume $\Gamma_{i j, k l}$ in the natural orbital basis as an explicit functional of $\left\{n_{i}\right\}$ only, for example, depending on $\sqrt{n_{i}}$ [2-5], or more general powers, $n_{i}^{\alpha}$ with $\alpha \approx 0.656$ [6,7]. Although simple in form, these functionals have been successful in describing both

*jwang572@hotmail.com

†KnowlesPJ@Cardiff.ac.uk molecular and condensed-phase electronic structure, and offer the potential to systematically advance beyond the ubiquitous Kohn-Sham density-functional framework.

In this paper, we demonstrate by counterexample that such functionals of $\Gamma_{i j, k l}$, based on occupation numbers alone, cannot be generally correct. The demonstration has implications for the design of improved descriptions of electronic structure in both free molecules and condensed phases; it also provides new insight on assessing the validity of existing functionals.

The square-root functional is potentially exact in the two-electron system [8] provided that one can overcome the problem of phase uncertainty, coming from the coefficients of wave-function expansion in terms of the natural orbitals, $c_{i}= \pm \sqrt{n_{i}}$. It is typically the case that all other phases take the opposite sign with respect to the dominant configuration in $\mathrm{H}_{2}$ when the bond length $R_{2}$ is small [9]. As $R_{2} \rightarrow \infty$, the phase pattern changes to half positive and half negative $[10,11]$. A functional of one-matrix reproducing the correct phases in all case is still missing. One may take the phase as additional variables [12], then this will go beyond Gilbert's original variable set: the natural orbitals and their occupation numbers. The two-matrix $\Gamma_{i j, k l}$ has some known properties that approximations to it should satisfy. Since it is the expectation value of the operator $a_{i}^{\dagger} a_{j}^{\dagger} a_{l} a_{k}$, where $\left\{a_{i}\right\}$ are the annihilation operators, it is both Hermitian and antisymmetric via the Pauli principle, $\Gamma_{i j, k l}=-\Gamma_{j i, k l}$. In the natural orbital basis, the partial trace of two-matrix reduces to

$$
\sum_{j} \Gamma_{i j, k j}=(N-1) n_{i} \delta_{i k}
$$

where $N$ is the number of electrons in the system. Most functionals $[2,3,7]$ fail to satisfy these requirements; such failure can be viewed as the introduction of unphysical self interaction, or more generally as undesirable departure from $N$-representability of the two-matrix.

In case of single determinant wave function or Hartree-Fock approximation, $n_{i}=1$ for each orbital. When the number of orbitals increases, some are more likely to occupy than others, so the eigenvalue spectrum of one-matrix can be taken as a measure of electron correlation [13,14]. The occupation numbers are constrained by $0 \leqslant n_{i} \leqslant 1$ and $\sum_{i} n_{i}=N$ due to the normalization requirement of the wave function [15]. The 
antisymmetrical requirement of the wave function imposes additional constraints on the one-matrix. A clear example is that of three electrons in six orbitals [16,17], where the six eigenvalues of one-matrix in nonincreasing order satisfy additional conditions: $n_{1}+n_{6}=1, n_{2}+n_{5}=1, n_{3}+n_{4}=1$, and $n_{5}+n_{6}-n_{4} \geqslant 0$. These lesser-known conditions are the $N$-representability conditions for the one-matrix, which sensitively depend on the system, especially the number of electrons and the number of orbitals [18]. The occupation numbers $n_{i}$ have the tendency to pin to these $N$-representability conditions (or the generalized Pauli constraints) [19-21]. It is difficult to build all known $N$-representability conditions into a functional model, but one can always get $N$-representable density matrices from any available accurate wave function to compare with those implicit in any given functional.

Our purpose is to highlight that there are systems having the same one-matrix eigenvalue spectrum but different two-matrix spectrum; a functional for $\Gamma_{i j, k l}$ based on $\left\{n_{i}\right\}$ only is thus nonunique. For three-electron systems, the one-matrix and two-matrix are always isospectral [22], so we investigate four-electron cases. Two systems are selected, the first is the hydrogen molecule $\mathrm{H}_{2}$ with a bond length $R_{2}$, together with two $\mathrm{H}$ atoms placed very far away; the second system is four $\mathrm{H}$ atoms placed on the corners of a square with side length $R_{4}$. In both cases, the ground-state singlet wave function is calculated. For illustrative purposes, we choose a minimal basis set (STO-3G), so that each system has four spatial orbitals or eight spin orbitals. The first system has two completely uncoupled electron pairs and can therefore be treated exactly by any method that is extensive and that is exact for two electrons, whereas the square-planar $\mathrm{H}_{4}$ has frustrated covalent bonds and contains true static electron correlation. For both systems, a closed-shell single-determinant description is far from the exact ground-state wave function. Comparison of these models is expected to shed light on the approximate electronic structure theories.

For the $D_{4 h}$ square-planar $\mathrm{H}_{4}$ system, the normalized ${ }^{1} B_{1 g}$ ground-state wave function can be expressed in terms of Slater determinants built from the four symmetry-adapted orbitals [23]: $a_{1 g}, e_{u x}, e_{u y}$, and $b_{2 g}$,

$$
\begin{aligned}
\Psi_{4}= & c_{1}\left(\left|a_{1 g}^{2} e_{u x}^{2}\right|-\left|a_{1 g}^{2} e_{u y}^{2}\right|\right) \\
& +c_{2}\left(\left|b_{2 g}^{2} e_{u x}^{2}\right|-\left|b_{2 g}^{2} e_{u y}^{2}\right|\right) \\
& +c_{3} \mid a_{1 g} e_{u x} e_{u y} b_{2 g} \frac{1}{2 \sqrt{3}}[\alpha \alpha \beta \beta+\beta \beta \alpha \alpha \\
& +\alpha \beta \alpha \beta+\beta \alpha \beta \alpha-2(\alpha \beta \beta \alpha+\beta \alpha \alpha \beta)] \mid,
\end{aligned}
$$

with $c_{3}=\sqrt{1-2 c_{1}^{2}-2 c_{2}^{2}}$. The eigenvalues of the one-matrix are then, for $\sigma=\alpha$ or $\beta$,

$$
\begin{gathered}
n_{a_{1 g}^{\sigma}}=\frac{1}{2}+c_{1}^{2}-c_{2}^{2}, \\
n_{b_{2 g}^{\sigma}}=1-n_{a_{1 g}^{\sigma}}, \\
n_{e_{u x}^{\sigma}}=n_{e_{u y}^{\sigma}}=\frac{1}{2} .
\end{gathered}
$$

For the $\mathrm{H}_{2}+2 \mathrm{H}$ system, in terms of the molecular $\sigma_{g}, \sigma_{u}$ and isolated-atom $A, B$ orbitals, the wave function is

$$
\Psi_{2}=\left|\left(c_{g} \sigma_{g}^{2}+c_{u} \sigma_{u}^{2}\right) A B \sqrt{\frac{1}{2}}(\alpha \beta-\beta \alpha)\right|,
$$

with $c_{u}=-\sqrt{1-c_{g}^{2}}$, and the one-matrix eigenvalues are

$$
\begin{gathered}
n_{\sigma_{g}^{\sigma}}=c_{g}^{2}, \\
n_{\sigma_{u}^{\sigma}}=1-n_{\sigma_{g}^{\sigma}}, \\
n_{A^{\sigma}}=n_{B^{\sigma}}=\frac{1}{2} .
\end{gathered}
$$

For both systems, the degenerate eigenvalue is $\frac{1}{2}$, and this leaves only one independent eigenvalue since the sum of onematrix eigenvalues from both spins should be $N=4$. Both $n_{\sigma_{g}^{\sigma}}$ and $n_{a_{1 g}^{\sigma}}$ approach $\frac{1}{2}$ monotonically from above as the atoms are separated, and one can then easily tune either $R_{2}$ or $R_{4}$ to make the one-matrix eigenvalues equal for both systems; this occurs whenever $c_{g}^{2}=\frac{1}{2}+c_{1}^{2}-c_{2}^{2}$. For example, at $R_{4}=2.0 \AA, c_{1}=$ $0.5833788, c_{2}=-0.247637, n_{a_{1 g}^{\alpha}}=0.779007$, and at $R_{2}=$ $1.79326407 \AA, c_{g}=0.882614, n_{\sigma_{g}^{\alpha}}=0.779007$.

For the above two wave functions, one may calculate $\Gamma_{i j, k l}$ as the expectation value of the operator $a_{i}^{\dagger} a_{j}^{\dagger} a_{l} a_{k}$. The nonredundant same spin block of $\Gamma_{i j, k l}$, with $i<j, k<l$, is

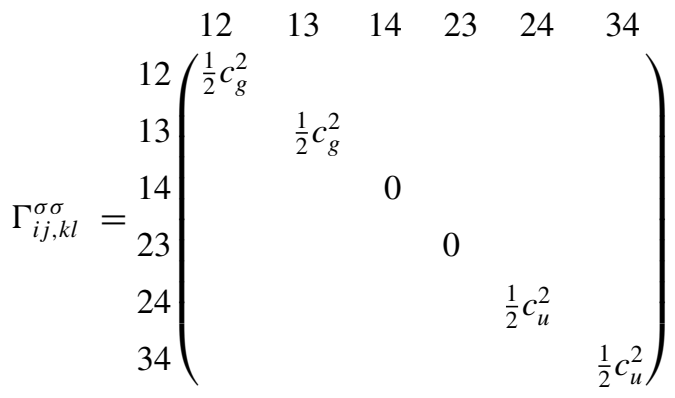

for the $\mathrm{H}_{2}+2 \mathrm{H}$ system, and for the $4 \mathrm{H}$ square system,

$$
\begin{aligned}
& \Gamma_{i j, k l}^{\sigma \sigma}=
\end{aligned}
$$

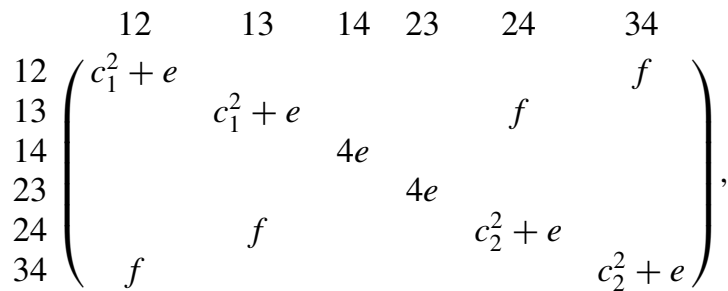

where $e=\frac{1}{12} c_{3}^{2}$ and $f=\frac{1}{2 \sqrt{3}}\left(c_{2}-c_{1}\right) c_{3}$. The labeling above and on the left of the matrix shows the the indices $i j, k l$, with the orbitals ordered as $\sigma_{g}, A, B, \sigma_{u}$ $\left(\mathrm{H}_{2}+2 \mathrm{H}\right)$ and $a_{1 g}, e_{u x}, e_{u y}, b_{2 g}\left(\mathrm{H}_{4}\right)$. For the $\mathrm{H}_{2}+2 \mathrm{H}$ 
system, the opposite-spin block of $\Gamma_{i j, k l}$ is

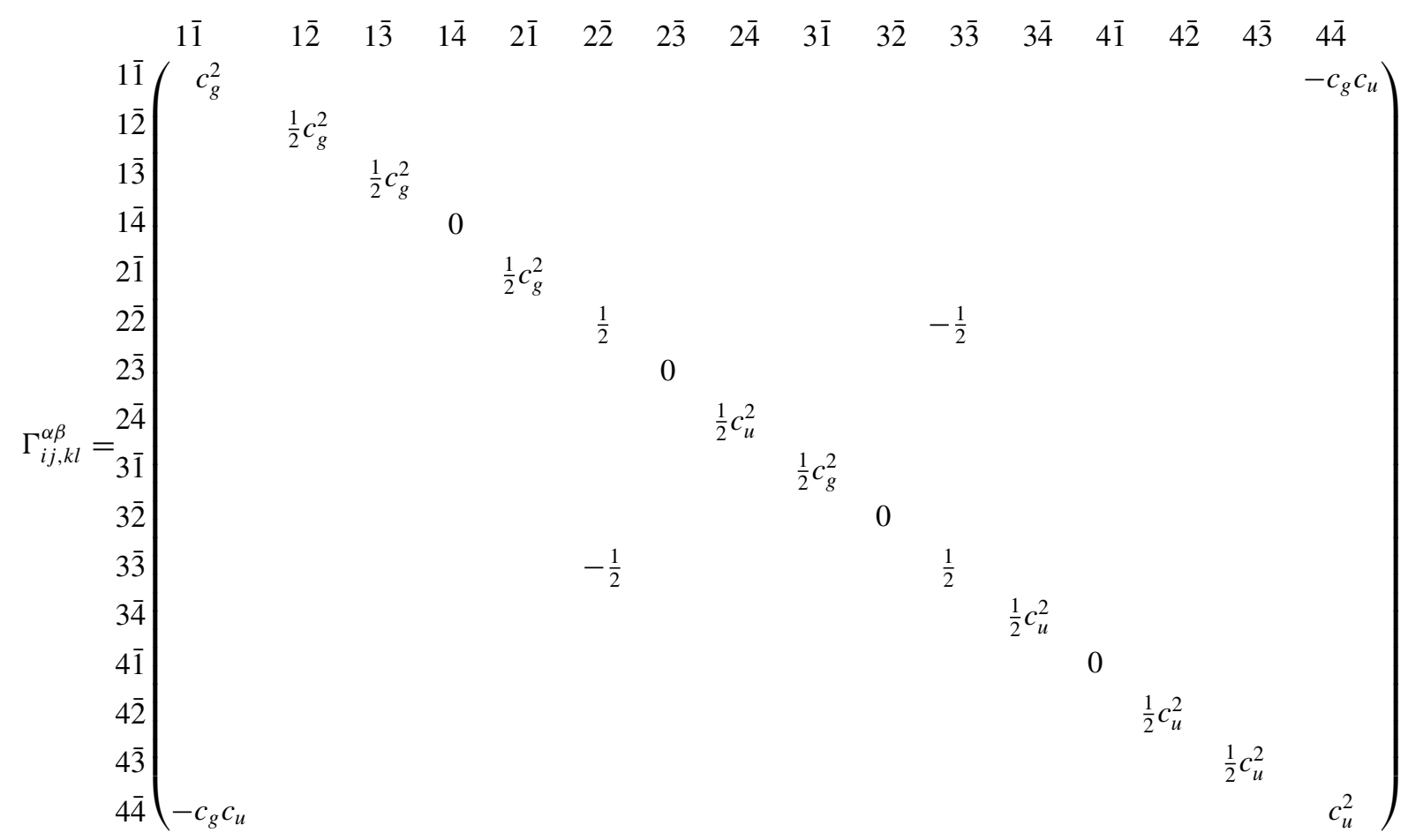

and for the $\mathrm{H}_{4}$ square system,

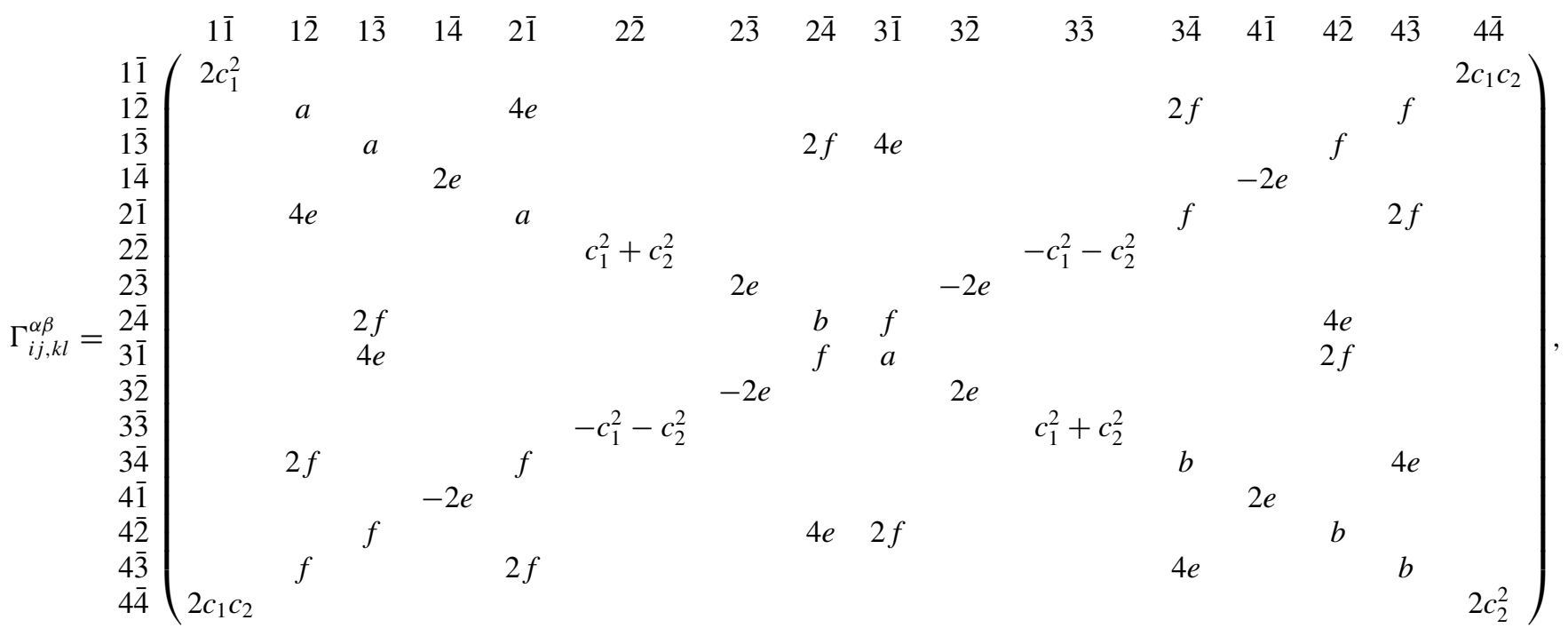

where $e=\frac{1}{12} c_{3}^{2}, a=c_{1}^{2}+5 e, b=c_{2}^{2}+5 e$, and $f=\frac{1}{2 \sqrt{3}}$ $\left(c_{2}-c_{1}\right) c_{3}$. Here, the indices with bars indicate $\beta$-spin orbitals.

The matrices are quite sparse, especially all the elements $\Gamma_{i j, k j}=0$ when $i \neq k$, and one can easily verify the conditions set by Eq. (4). Algebraic one-matrix functionals are typically two-index JK type [24], i.e., only elements with indices $(i j, i j)$ and $(i j, j i)$ are included. Allowing complex orbitals, JKL-type functional is also possible with only two indices, where $(i j, j i)$ elements will be different from that of $(i i, j j)$ [25,26]. For the square $\mathrm{H}_{4}$ system, there are additional off-diagonal elements such as $\Gamma_{12,34}, \Gamma_{12}, 3 \overline{4}$. Those elements are caused by excitations from or to the configuration involving $a_{1 g} e_{u x} e_{u y} b_{2 g}$, i.e., the $c_{3}$ term in Eq. (5). Those elements with four different indices contribute to the correlation energy, but they are missing in $J K$-type functionals; however, those functionals include different factors than the exact two-matrix for the $J$ and $K$ integrals, and these can be considered to partly compensate for the missing contributions. Some of this compensation can be understood for this example by considering the limit $R_{4} \rightarrow \infty$. One can show that the simple square-root functional [8] produces the exact energy, including cancelation of all long-range $R_{4}^{-1}$ contributions, in the regime where the atoms no longer overlap, despite the fact that the two-matrix from the functional is completely different to that of the exact ground state. 
TABLE I. Eigenvalues of the two-matrix for the $\mathrm{H}_{2}+2 \mathrm{H}$ and $\mathrm{H}_{4}$ models. Geometries: $R_{2}=1.79326407 \AA, R_{4}=2.0 \AA$. The numbers in parentheses are the degeneracies.

\begin{tabular}{cc}
\hline \hline $\mathrm{H}_{2}+2 \mathrm{H}$ & $\mathrm{H}_{4}$ \\
\hline $1.0000000000(2)$ & $0.8033100415(2)$ \\
$0.3895033775(8)$ & $0.6966765951(2)$ \\
$0.1104966225(8)$ & $0.3926620486(6)$ \\
$0.0000000000(10)$ & $0.0655633195(6)$ \\
& $0.0417746319(6)$ \\
& $0.0000133634(2)$ \\
& $0.0000000000(4)$ \\
\hline \hline
\end{tabular}

Table I displays the eigenvalue spectra of the two-matrix $\Gamma_{i j, k l}$, normalized to $N(N-1) / 2=6$, for the two model systems. These quantities are invariant to orbital rotations and serve to demonstrate further that the two-matrices are inherently different for the two systems. One effect of the off-diagonal elements in the square $\mathrm{H}_{4}$ system is to lift some of the degeneracy in the eigenvalues. For example, the off-diagonal elements in the $\Gamma_{i j, k l}^{\alpha \beta}$ block,

$$
\begin{aligned}
& 2 \overline{1} \\
& 3 \overline{4}
\end{aligned}\left(\begin{array}{cccc}
1 \overline{2} & 2 \overline{1} & 3 \overline{4} & 4 \overline{3} \\
a & 4 e & 2 f & f \\
4 e & a & f & 2 f \\
2 f & f & b & 4 e \\
f & 2 f & 4 e & b
\end{array}\right),
$$

lift the degeneracy in $a$ and $b$ when $e \neq 0$ or $f \neq 0$. For $R_{4}=2.0 \AA$, this block leads to the eigenvalues 0.6966765951 , $0.3926620486,0.0417746319$, and 0.0000133634 shown in Table I.

One may easily find many other possible pairs of $R_{2}$ and $R_{4}$ resulting in the same one-matrix eigenvalue spectrum, but different two-matrix $\Gamma_{i j, k l}$. This can be true for any type of basis function (Gaussian type, or Slater type, or numerical type [27]) as long as there are eight spin orbtials. This throws into question the reliability of a model for $\Gamma_{i j, k l}$-based on only the one-matrix eigenvalues, which will give identical two-matrix for the models presented above. The problem stems from the fact that the mapping from the one-matrix eigenvalues to the two-matrix is one-to-many-there is no unique two-matrix that can be constructed using a simple algebraic functional of the orbital occupation numbers.

These findings do not challenge the general result, via the Hohenberg-Kohn and Gilbert theorems [1,28,29], that the total energy is a universal upper-bound functional of the one-matrix; i.e., both the natural orbitals and their occupation numbers should be taken as functional variables. Those theorems, however, do not require the density-matrix functional to have an easily computable algebraic form. The findings are of relevance for any situation where covalent bonds are being broken or partially broken; this includes many reaction transition states, and the computation of vibrational frequencies.

Equation (10) implies the square-root functional, the $\mathrm{H}_{2}+$ $2 \mathrm{H}$ system is thus essentially a two-electron correlated system. For the square $\mathrm{H}_{4}$ system, Eq. (6) indicates that there are two independent variables in the wave function: $c_{1}$ and $c_{2}$, but only one variable in the one-matrix eigenvalue spectrum. So the information of the one-matrix spectrum is not enough to fix the wave function. Functional based on the one-matrix eigenvalues thus cannot generate a $N$-representable two-matrix for the square $\mathrm{H}_{4}$ system. The origin of the one-to-many mapping can also be understood through the contraction relation, Eq. (4), since contraction of different sets of two-matrix elements can easily result in the same one-matrix. Since the three-index and four-index elements of $\Gamma$ are neglected in the two-index JKor JKL-type functional, one cannot remedy its representation deficiency simply by increasing the number of basis functions.

For the wave function of superconductor, Yang [30,31] found the one-matrix eigenvalue spectrum is trivially uniform with its value subject to the normalization condition $\sum_{i} n_{i}=$ $N$, while the spectra for the two-matrix is highly structured and dominated by a single eigenvalue. This demonstrates that the one-matrix eigenvalues alone are unable to convey certain information in the two-matrix or the wave function

In principle, one can always start from an approximate form of wave function, use the coefficients of the wave-function expansion as the parameters, and express the two-matrix elements to obtain a parameterized energy functional, as we have demonstrated here. Previous examples of such a strategy include the direct use of geminals [32-35], the approximation of the variational energy expression using the coupled cluster method [36] or configuration interaction method [37], and the full configuration interaction Monte Carlo method [38]. An advantage of such an approach is that $N$-representability is considered from the outset.

As well as providing a framework for the analysis of one-matrix functionals, the result also can serve for the evaluation of other theoretical methods based on one-matrix and/or two-matrix, such as density-cumulant functional theory [39], intracule functional models [40], and the density-matrix approach based on the $N$-representability conditions [41-43]. Our result suggests that the correlation measures [13] based on the one-matrix eigenvalue spectrum have to be viewed critically: the von Neumann entropy, $S=-\sum_{i} n_{i} \ln n_{i}$, does not distinguish between the very different electron correlation effects of the two model systems we have considered. Alternative measures for electron correlation have been discussed in Refs. [44,45], and our result suggests that measures based on the the eigenvalue spectrum of the two-matrix are likely to be more successful than those that depend only on the one-matrix.

In summary, two model systems with equal one-matrix eigenvalues but different two-matrix are presented, the examples lead automatically to the question on the uniquesness for algebraic one-matrix functional or correlation measure based solely on the eigenvalues of one-matrix. In Gilbert's original theorem, the functional variable is the one-matrix; i.e., both the eigenvalues and eigenvectors of one-matrix should be taken into account. Reduction of functional variables to only the eigenvalues shrinks the variational space of the functional, which causes the representation problem.

We acknowledge discussions with S. Leoni and E. J. Baerends. J.W. is supported by National Natural Science Foundation of China Grant No. 11274109. 
[1] M. Levy, Proc. Natl. Acad. Sci. USA 76, 6062 (1979).

[2] A. M. K. Müller, Phys. Lett. A 105, 446 (1984).

[3] S. Goedecker and C. J. Umrigar, Phys. Rev. Lett. 81, 866 (1998).

[4] D. R. Rohr, K. Pernal, O. V. Gritsenko, and E. J. Baerends, J. Chem. Phys. 129, 164105 (2008).

[5] M. Piris, Int. J. Quantum Chem. 113, 620 (2013).

[6] S. Sharma, J. K. Dewhurst, N. N. Lathiotakis, and E. K. U. Gross, Phys. Rev. B 78, 201103 (2008).

[7] S. Sharma, J. K. Dewhurst, S. Shallcross, and E. K. U. Gross, Phys. Rev. Lett. 110, 116403 (2013).

[8] P.-O. Löwdin and H. Shull, Phys. Rev. 101, 1730 (1956).

[9] S. Goedecker and C. J. Umrigar, in Many-Electron Densities and Reduced Density Matrices, edited by J. Cioslowski (Kluwer Academic, Dordrecht/New York, 2000), Chap. 8, pp. 165-181.

[10] J. Cioslowski and K. Pernal, Chem. Phys. Lett. 430, 188 (2006).

[11] X. W. Sheng, Ł. M. Mentel, O. V. Gritsenko, and E. J. Baerends, J. Chem. Phys. 138, 164105 (2013).

[12] K. J. H. Giesbertz, O. V. Gritsenko, and E. J. Baerends, Phys. Rev. Lett. 105, 013002 (2010).

[13] G. T. Smith, H. L. Schmider, and V. H. Smith, Jr., Phys. Rev. A 65, 032508 (2002).

[14] P. Ziesche, V. H. Smith, Jr., M. Hô, S. P. Rudin, P. Gersdorf, and M. Taut, J. Chem. Phys. 110, 6135 (1999).

[15] P.-O. Löwdin, Phys. Rev. 97, 1474 (1955).

[16] R. E. Borland and K. Dennis, J. Phys. B: Atom. Molec. Phys. 5, 7 (1972).

[17] M. B. Ruskai, J. Phys. A: Math. Theor. 40, F961 (2007).

[18] M. Altunbulak and A. Klyachko, Commun. Math. Phys. 282, 287 (2008).

[19] A. A. Klyachko, arXiv:0904.2009.

[20] C. Schilling, D. Gross, and M. Christandl, Phys. Rev. Lett. 110, 040404 (2013).

[21] R. Chakraborty and D. A. Mazziotti, Phys. Rev. A 89, 042505 (2014).
[22] B. Carlson and J. Keller, Phys. Rev. 121, 659 (1961).

[23] J. Paldus, P. Piecuch, L. Pylypow, and B. Jeziorski, Phys. Rev. A 47, 2738 (1993).

[24] J. Cioslowski, M. Buchowiecki, and P. Ziesche, J. Chem. Phys. 119, 11570 (2003).

[25] R. van Meer, O. V. Gritsenko, and E. J. Baerends, J. Chem. Phys. 140, 024101 (2014).

[26] Ł. M. Mentel, R. van Meer, O. V. Gritsenko, and E. J. Baerends, J. Chem. Phys. 140, 214105 (2014).

[27] A. D. Becke, J. Chem. Phys. 88, 2547 (1988).

[28] P. Hohenberg and W. Kohn, Phys. Rev. 136, B864 (1964).

[29] T. Gilbert, Phys. Rev. B 12, 2111 (1975).

[30] C. Yang, Rev. Mod. Phys. 34, 694 (1962).

[31] A. J. Coleman, J. Math. Phys. 6, 1425 (1965).

[32] W. Kutzelnigg, J. Chem. Phys. 40, 3640 (1964).

[33] D. A. Mazziotti, Chem. Phys. Lett. 338, 323 (2001).

[34] V. A. Rassolov, J. Chem. Phys. 117, 5978 (2002).

[35] E. Pastorczak and K. Pernal, Phys. Chem. Chem. Phys. 17, 8622 (2015).

[36] J. B. Robinson and P. J. Knowles, J. Chem. Phys. 136, 054114 (2012).

[37] C. Kollmar, J. Chem. Phys. 125, 084108 (2006).

[38] G. H. Booth, A. J. W. Thom, and A. Alavi, J. Chem. Phys. 131, 054106 (2009).

[39] W. Kutzelnigg, J. Chem. Phys. 125, 171101 (2006).

[40] P. M. W. Gill, Annu. Rep. Prog. Chem. Sect. C: Phys. Chem. 107, 229 (2011).

[41] H. Nakatsuji and K. Yasuda, Phys. Rev. Lett. 76, 1039 (1996).

[42] D. A. Mazziotti, Phys. Rev. Lett. 101, 253002 (2008).

[43] C. Valdemoro, D. R. Alcoba, L. M. Tel, and E. Prez-Romero, Int. J. Quantum Chem. 109, 2622 (2009).

[44] A. D. Gottlieb and N. J. Mauser, Phys. Rev. Lett. 95, 123003 (2005).

[45] T. Juhász and D. A. Mazziotti, J. Chem. Phys. 125, 174105 (2006). 